\title{
A representation learning model based on variational inference and graph autoencoder for predicting IncRNA-disease associations
}

\author{
Zhuangwei Shi ${ }^{1}$, Han Zhang ${ }^{1 *}$, Chen Jin $^{2}$, Xiongwen Quan ${ }^{1}$ and Yanbin Yin ${ }^{3}$
}

\author{
*Correspondence: \\ zhanghan@nankai.edu.cn \\ ${ }^{1}$ College of Artificial \\ Intelligence, Nankai \\ University, Tongyan Road, \\ 300350 Tianjin, China \\ Full list of author information \\ is available at the end of the \\ article
}

\begin{abstract}
Background: Numerous studies have demonstrated that long non-coding RNAs are related to plenty of human diseases. Therefore, it is crucial to predict potential IncRNAdisease associations for disease prognosis, diagnosis and therapy. Dozens of machine learning and deep learning algorithms have been adopted to this problem, yet it is still challenging to learn efficient low-dimensional representations from high-dimensional features of IncRNAs and diseases to predict unknown IncRNA-disease associations accurately.

Results: We proposed an end-to-end model, VGAELDA, which integrates variational inference and graph autoencoders for IncRNA-disease associations prediction. VGAELDA contains two kinds of graph autoencoders. Variational graph autoencoders (VGAE) infer representations from features of IncRNAs and diseases respectively, while graph autoencoders propagate labels via known IncRNA-disease associations. These two kinds of autoencoders are trained alternately by adopting variational expectation maximization algorithm. The integration of both the VGAE for graph representation learning, and the alternate training via variational inference, strengthens the capability of VGAELDA to capture efficient low-dimensional representations from high-dimensional features, and hence promotes the robustness and preciseness for predicting unknown IncRNA-disease associations. Further analysis illuminates that the designed co-training framework of IncRNA and disease for VGAELDA solves a geometric matrix completion problem for capturing efficient low-dimensional representations via a deep learning approach.
\end{abstract}

Conclusion: Cross validations and numerical experiments illustrate that VGAELDA outperforms the current state-of-the-art methods in IncRNA-disease association prediction. Case studies indicate that VGAELDA is capable of detecting potential IncRNAdisease associations. The source code and data are available at https://github.com/ zhanglabNKUNGAELDA.

Keywords: Variational inference, Graph autoencoder, IncRNA-disease association, Representation learning author(s) and the source, provide a link to the Creative Commons licence, and indicate if changes were made. The images or other third party material in this article are included in the article's Creative Commons licence, unless indicated otherwise in a credit line to the material. If material is not included in the article's Creative Commons licence and your intended use is not permitted by statutory regulation or exceeds the permitted use, you will need to obtain permission directly from the copyright holder. To view a copy of this licence, visit http:// creativecommons.org/licenses/by/4.0/. The Creative Commons Public Domain Dedication waiver (http://creativecommons.org/publi cdomain/zero/1.0/) applies to the data made available in this article, unless otherwise stated in a credit line to the data. 


\section{Introduction}

LncRNAs are RNAs longer than 200 nucleotides thus losing the function of encoding, while they can still influence a series of biological processes, such as gene transcription, cell apoptosis, hormonal regulation, and immune response. Hence, lncRNAs are closely linked to plenty of human diseases [1-3]. For instance, lncRNA PANDAR is a novel biomarker of breast cancer, which upregulates proliferation of breast cancer cells [4]. Sun et al. [5] found that the downregulation of lncRNA MEG3 promotes proliferation of gastric cancer cells. Faghihi et al. [6] reported that lncRNA BACE1AS can regulate mRNA BACE1, while BACE1 is associated with the generation of beta-amyloid, which can cause Alzheimer's disease. Therefore, it is essential to predict potential lncRNA-disease associations for disease prevention, detection, diagnosis and treatment. However, there are only a small number of lncRNA-disease associations that have been discovered so far, and it would be ideal to predict more potential lncRNA-disease associations using computational approaches. Generally, computational methods, especially machine learning algorithms, are more time-efficient and cost-effective to detect potential lncRNA-disease associations compared with experimental methods.

Previous machine learning approaches for predicting lncRNA-disease associations can be categorized into three types. The first type of methods is based on matrix analysis. Two commonly used matrix analysis methods for predicting lncRNA-disease associations are manifold regularization [7] and matrix completion [8], which suggest that lncRNA-disease association matrix follow manifold constraint or low-rank constraint, respectively. Manifold regularization based methods have been widely adopted for link prediction of biological entities [9-11]. Laplacian regularized least square (LRLS) method [7] integrates manifold regularization and basic least square method. Chen and Yan [12] proposed LRLSLDA that applied LRLS to the lncRNA-disease associations prediction, after the construction of an IncRNA graph and a disease graph through computing feature similarity respectively. Based on LRLSLDA, several methods were proposed to improve the performance of LRLS by integrating different types of feature similarities $[13,14]$. In addition, lncRNA-disease associations can be viewed as links on an lncRNAdisease bipartite graph. Matrix completion algorithm [8] can solve link prediction problem by applying low-rank constraint to association matrix, and have been commonly applied to forecast associations among biological entities [15-17]. Lu et al. [18] proposed a matrix completion based method for predicting lncRNA-disease associations. Geometric matrix completion $[19,20]$ incorporates manifold regularization into the matrix completion problem, and Lu et al. [21] proposed a geometric matrix completion based framework for predicting lncRNA-disease associations.

The second type of methods focuses on the integration of heterogeneous features. Applying multi-source features to learn better representations is an efficient technique for predicting associations among biological entities [22, 23]. Lan et al. [24] developed a web server for lncRNA-disease association prediction by integrating multiple features of lncRNAs and diseases to construct lncRNA similarity network and disease similarity network. Fu et al. [25] integrated heterogeneous data for lncRNA-disease associations prediction by matrix factorization with low-rank constraint. Ding et al. [26] inferred links on lncRNA-disease bipartite graph via 
lncRNA-disease-gene tripartite graph. Yao et al. [27] adopted random forest for feature selection in lncRNA-disease associations prediction.

The third type is deep learning approaches. Neural networks are competent to capture efficient low-dimensional representations from high-dimensional features of biological entities, and deep learning based methods were proposed for detecting potentional associations among biological entities [17, 22, 28]. Thus, several deep learning models applying autoencoders for representation learning of lncRNA features and disease features were proposed [29, 30]. Graph neural networks (GNN) [31] were proposed in deep learning on graphs. Hence, there are some recent approaches for lncRNA-disease associations prediction based on GNN. Xuan et al. [32] integrated graph convolutional networks (GCN) [33] and CNN to learn representations from features of lncRNAs and diseases. GCN is applicable for link prediction on bipartite graph [34], and Wu et al. [35] adopted graph autoencoder to predict lncRNA-disease associations on lncRNA-disease bipartite graph.

In this paper, we proposed a method, VGAELDA, that integrates variational inference and graph autoencoders to improve the performance of IncRNA-disease associations prediction. In previous works, feature inference and label propagation are two separated stages in these methods, and hence label propagation procedure may fail to make the full use of low-dimensional representations learned from highdimensional features. Using deep learning approaches, our method proposed an end-to-end framework, which fuses feature inference and label propagation under the variational inference algorithm of Graph Markov Neural Networks (GMNN) [36]. Specifically, the feature inference network in VGAELDA is designed as a variational graph autoencoder (VGAE) [37] that learns representations from feature matrices of lncRNAs and diseases respectively. Furthermore, the label propagation network in our model is a graph autoencoder (GAE) [37] that estimates the score of unknown lncRNA-disease pairs from known ones. These two graph autoencoders learn from feature and propagate label alternately, which are trained by variational EM algorithm, and are implemented as a representation learning framework. This framework minimizes the difference of the representations learned by two autoencoders respectively. Therefore, VGAELDA has the following advantages. (i) VGAE is preferable to infer low-dimensional representations from high-dimensional features in a graph, and these representations can better depict similarities and dependencies among nodes. This would significantly enhance the robustness and preciseness of prediction without handcrafted feature similarities. (ii) VGAELDA implements the variational EM algorithm as a representation learning framework, by training the feature inference autoencoder and the label propagation autoencoder alternately. (iii) VGAELDA provides a useful solution to the geometric matrix completion problem via deep learning, because autoencoders tend to minimize the rank of outputs, and we suggest that manifold regularization can be obtained via the alternate training of two graph autoencoders. (iv) VGAELDA implements an efficient way to integrate information from lncRNA space and disease space. Experiments illustrate that VGAELDA is superior to the current state-of-the-art methods, and case studies on several diseases illustrate the capability of VGAELDA to detect new IncRNA-disease associations. 


\section{Results}

\section{Datasets}

In this paper, we adopted two datasets for evaluation. Dataset1 is an lncRNA-disease association dataset from [26], including 540 associations among 115 lncRNAs and 178 diseases. Dataset2 is an lncRNA-disease association dataset from [25], including 2697 associations among 240 lncRNAs and 412 diseases. Both of them were collected from LncRNADisease [38] Database.

For each lncRNA, we adopted Word2Vec to compute the feature vector. Word2Vec [39] is an efficient method to learn the embedding vectors of natural language, and BioVec [40] (https://pypi.org/project/biovec/) applied Word2Vec for representation learning of biological sequences, including protein sequences or nucleotide sequences. In VGAELDA, the length of each vector was set at 300. We downloaded lncRNA sequences from the Nucleotide Database of NCBI.

For each disease, we adopted its associations with 1415 genes as the feature vector on Dataset1. Dataset2 includes disease associated with 15527 genes. After removing genes that are not associated with any diseases, 10146 genes remain and are used as the feature vector on Dataset2. Information with respect to diseases was collected from DisGeNet [41] and Disease Ontology [42].

\section{Comparison with other methods}

\section{Cross validation}

We compared our proposed method, VGAELDA, with other five state-of-the-art methods:

- LRLSLDA: Chen and Yan [12] proposed a Laplacian regularized least square (LRLS) method [7] based framework to predict lncRNA-disease associations.

- SIMCLDA: Lu et al. [18] proposed a computational method for predicting lncRNA-disease associations based on speedup inductive matrix completion (SIMC) [43].

- TPGLDA: Ding et al. [26] integrated heterogeneous features by constructing lncRNAdisease-gene tripartite graph for lncRNA-disease associations prediction.

- SKFLDA: Xie et al. [14] proposed SKFLDA that applied kernel fusion trick for different types of similarities to improve the preciseness of lncRNA-disease associations prediction.

- GAMCLDA: Wu et al. [35] implemented GAMCLDA, adopting graph autoencoders to predict lncRNA-disease associations on lncRNA-disease bipartite graph.

We adopted 5-fold cross validation to obtain the result, and the metrics were listed below.

$$
\begin{aligned}
& \text { Sensitivity }=\frac{T P}{T P+F N}=T P R=\text { Recall, } \\
& \text { Specificity }=\frac{T N}{T N+F P}=1-F P R,
\end{aligned}
$$




$$
\begin{aligned}
& \text { Accuracy }=\frac{T N+T P}{T N+T P+F N+F P}, \\
& \text { Precision }=\frac{T P}{T P+F P}, \\
& F 1=\frac{2 \times \text { Precision } \times \text { Recall }}{\text { Precision }+ \text { Recall }}, \\
& M c c=\frac{T P \times T N-F P \times F N}{\sqrt{(T P+F N) \times(T P+F P) \times(T N+F N) \times(T N+F P)}},
\end{aligned}
$$

where TP denotes true positive, FN denotes false negative, TN denotes true negative, FP denotes false negative, TPR denotes true positive rate, FPR denotes false positive rate, and Mcc denotes Matthews correlation coefficient. The receiver operating characteristic (ROC) curve can be plotted by TPR and FPR, while the area under ROC curve (AUROC) and the area under precision-recall curve (AUPR) are important metrics to measure the performance of a binary classification model.

We plotted the ROC curves and PR curves of Dataset 1 and Dataset 2 on Figs. 1 and 2 , respectively. We ran our experiments for 5 times, and the mean values and standard deviations of AUROC and AUPR are listed on Table 1. The AUROC and AUPR values of VGAELDA in 5 times are listed in Additional file 1.

The results show that VGAELDA outperforms the other five state-of-the-art methods in both AUROC and AUPR, on both datasets. Specifically, for the AUPR values obtained by other five state-of-the-art methods, GAMCLDA performs best in 5-fold CV on both Dataset1 and Dataset2, which gives AUPR values at 0.5794 and 0.3798 respectively. Compared with these AUPR values, VGAELDA significantly outperforms these previous methods by increasing the AUPR values $45 \%$ in 5 -fold $\mathrm{CV}$ on Dataset1, and 116\% in 5-fold CV on Dataset2.


Fig. 1 ROC and PR curves of different methods on Dataset1. In AUROC, VGAELDA (AUROC $=0.9680$ ) outperforms GAMCLDA (0.9299), SKFLDA (0.9154), TPGLDA (0.7936), SIMCLDA (0.8293) and LRLSLDA (0.8157). In AUPR, VGAELDA (AUPR = 0.8380) outperforms GAMCLDA (0.5794), SKFLDA (0.4024), TPGLDA (0.5308), SIMCLDA (0.5357) and LRLSLDA (0.2035) 

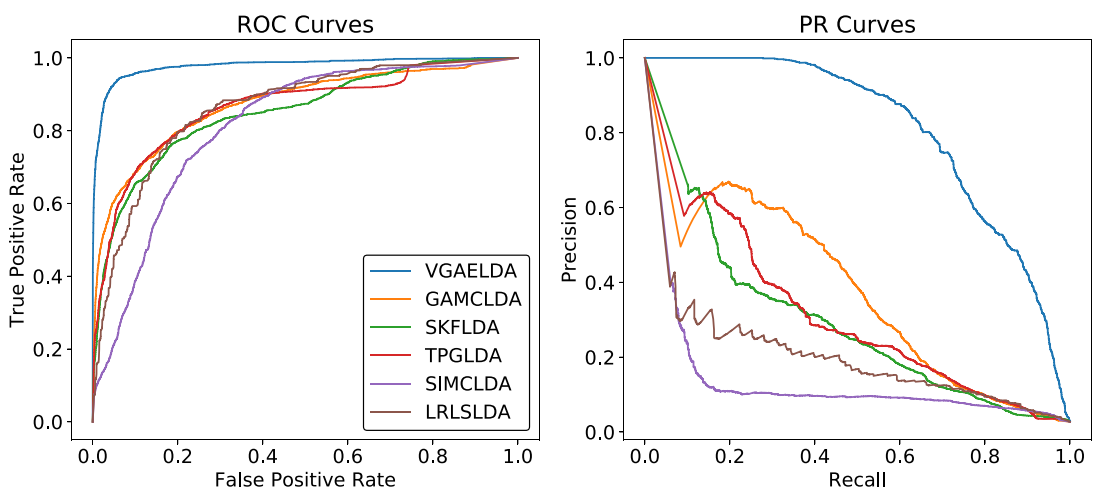

Fig. $2 R O C$ and PR curves of different methods on Dataset2. In AUROC, VGAELDA (AUROC $=0.9692$ ) outperforms GAMCLDA (0.8841), SKFLDA (0.8524), TPGLDA (0.8771), SIMCLDA (0.8146) and LRLSLDA (0.8627). In AUPR, VGAELDA (AUPR = 0.8203) outperforms GAMCLDA (0.3798), SKFLDA (0.2831), TPGLDA (0.3192), SIMCLDA (0.1189) and LRLSLDA (0.1812)

Table 1 Mean values and standard deviations of AUROC and AUPR on Dataset1 and Dataset2, compared with different methods

\begin{tabular}{llllll}
\hline \multirow{2}{*}{ Method } & \multicolumn{2}{l}{ Dataset1 } & & \multicolumn{2}{l}{ Dataset2 } \\
\cline { 2 - 3 } & AUROC & AUPR & & AUROC & AUPR \\
\hline LRLSLDA & $0.8157 \pm 0.0005$ & $0.2035 \pm 0.0001$ & & $0.8627 \pm 0.0017$ & $0.1812 \pm 0.0021$ \\
SIMCLDA & $0.8293 \pm 0.0023$ & $0.5357 \pm 0.0011$ & & $0.8146 \pm 0.0042$ & $0.1189 \pm 0.0076$ \\
TPGLDA & $0.7936 \pm 0.0054$ & $0.5308 \pm 0.0028$ & & $0.8771 \pm 0.0053$ & $0.3192 \pm 0.0058$ \\
SKFLDA & $0.9154 \pm 0.0013$ & $0.4024 \pm 0.0017$ & & $0.8524 \pm 0.0066$ & $0.2831 \pm 0.0085$ \\
GAMCLDA & $0.9299 \pm 0.0033$ & $0.5794 \pm 0.0143$ & & $0.8841 \pm 0.0110$ & $0.3798 \pm 0.0154$ \\
VGAELDA & $\mathbf{0 . 9 6 8 0} \pm 0.0042$ & $\mathbf{0 . 8 3 8 0} \pm 0.0041$ & & $\mathbf{0 . 9 6 9 2} \pm 0.0080$ & $\mathbf{0 . 8 2 0 3} \pm 0.0139$ \\
\hline
\end{tabular}

The bold number is the highest value of each column, which is achieved by our method, VGAELDA. The bold clarifies the superiority of our method

\section{Evaluation on imbalanced data}

As the datasets are imbalanced, i.e., the number of negative samples is far more than positive samples, it is essential to evaluate the capability to retrieve true positive samples from predicted positive ones. In our experiments, the evaluation was implemented through the following two ways. In summary, VGAELDA performs the best in both evaluation ways.

Firstly, we evaluated the performance of our model at high stringency level of specificity according to Eq. (23456). We fixed specificity at 0.95 and 0.99 , and then computed sensitivity, accuracy, precision, F1-score and Mcc. The results of Dataset1 and Dataset2 are listed on Additional file 2 and Table 2, respectively, which illustrate that VGAELDA outperforms other five methods at all five metrics, and in both datasets. Matthews correlation coefficient (Mcc) is a comprehensive metric in binary classification on imbalanced data [44]. For the Mcc values obtained by the other five state-of-the-art methods, SKFLDA performs the best at $S p=0.95$ on Dataset1, which obtains 0.4637, GAMCLDA performs the best at $S p=0.99$ on Dataset1 and both $S p=0.95$ and 0.99 on Dataset2, which obtains $0.5804,0.3855$ and 0.4860 respectively. VGAELDA outperforms these 
Table 2 Binary classification metrics of different methods on Dataset2

\begin{tabular}{lllllll}
\hline Sp & Method & Sn & Acc & Pre & F1 & Mcc \\
\hline 0.95 & LRLSLDA & 0.4572 & 0.9369 & 0.2051 & 0.2831 & 0.2777 \\
& SIMCLDA & 0.2128 & 0.9299 & 0.1066 & 0.1421 & 0.1169 \\
& TPGLDA & 0.5565 & 0.9394 & 0.2384 & 0.3338 & 0.3380 \\
& SKFLDA & 0.5284 & 0.9385 & 0.2286 & 0.3191 & 0.3206 \\
& GAMCLDA & 0.6377 & 0.9415 & 0.2635 & 0.3729 & 0.3855 \\
0.99 & VGAELDA & $\mathbf{0 . 9 3 2 9}$ & $\mathbf{0 . 9 4 9 5}$ & $\mathbf{0 . 3 4 3 4}$ & $\mathbf{0 . 5 0 2 0}$ & $\mathbf{0 . 5 4 9 0}$ \\
& LRLSLDA & 0.1591 & 0.9676 & 0.3145 & 0.2113 & 0.2086 \\
& SIMCLDA & 0.1020 & 0.9658 & 0.2223 & 0.1398 & 0.1348 \\
& TPGLDA & 0.2673 & 0.9703 & 0.4279 & 0.3291 & 0.3238 \\
& SKFLDA & 0.2354 & 0.9694 & 0.3976 & 0.2958 & 0.2913 \\
& GAMCLDA & 0.4472 & 0.9752 & 0.5558 & 0.4956 & 0.4860 \\
& VGAELDA & $\mathbf{0 . 7 8 3 1}$ & $\mathbf{0 . 9 8 4 3}$ & $\mathbf{0 . 6 8 6 8}$ & $\mathbf{0 . 7 3 1 8}$ & $\mathbf{0 . 7 2 5 4}$
\end{tabular}

The bold number is the highest value of each column, which is achieved by our method, VGAELDA. The bold clarifies the superiority of our method

Sp specificity, Sn sensitivity, Acc accuracy, Pre precision, F1 F1-score, Mcc Matthews correlation coefficient

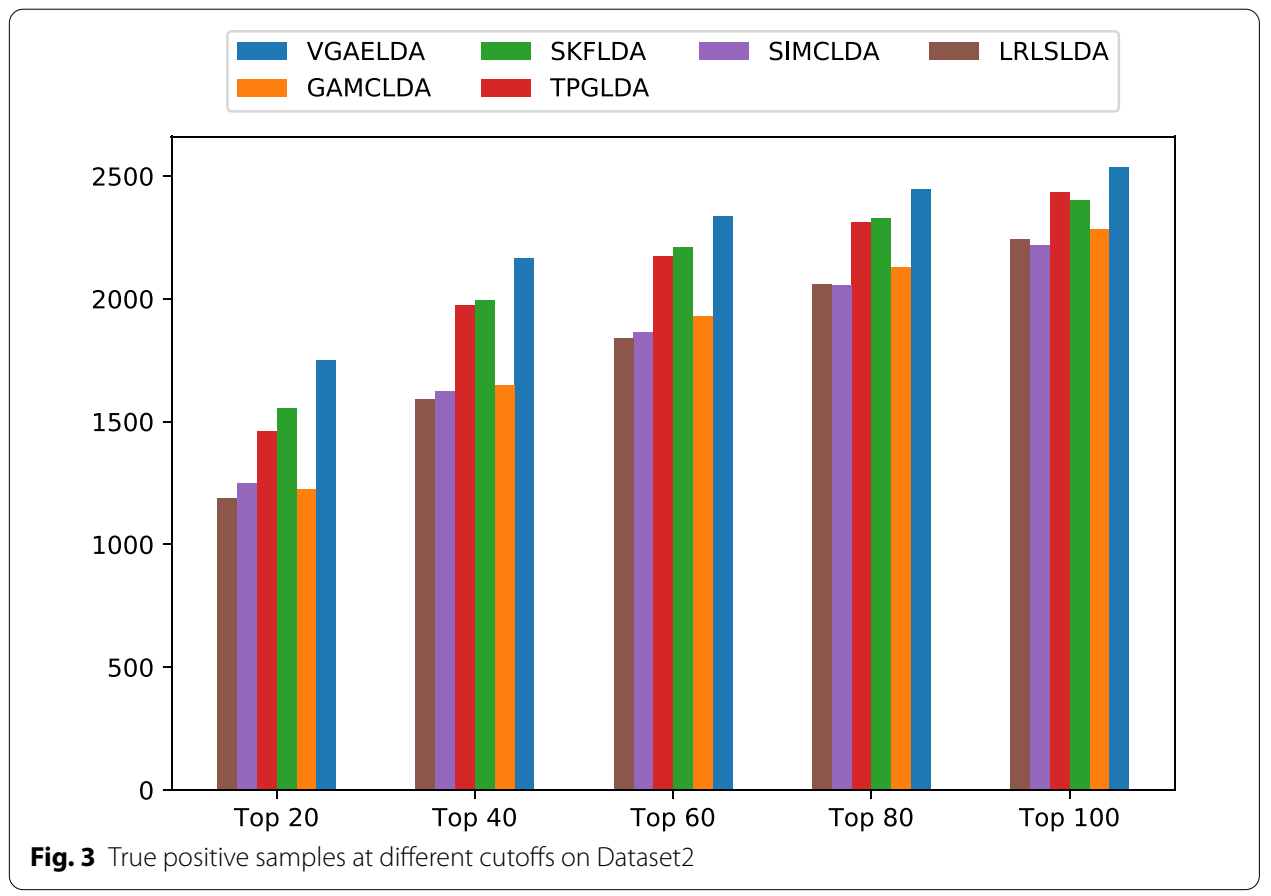

methods by improving the Mcc values $13 \%$ and $28 \%$ at $S p=0.95$ and 0.99 on Dataset1, and $42 \%$ and $49 \%$ at $S p=0.95$ and 0.99 on Dataset 2 .

Secondly, we evaluated recall score (i.e. sensitivity) via counting the number of true positive samples at different top- $k$ cutoffs, according to Eq. (1), where $k \in\{20,40,60,80,100\}$. The bar charts depicting the number of true positive samples at different top- $k$ cutoffs on Dataset1 and Dataset2 are shown on Additional file 3 and Fig. 3, respectively. VGAELDA retrieves the most true positive samples at all 5 cutoffs on both Dataset1 and Dataset2. 
Table 3 Top 10 predicted IncRNAs associated with breast cancer on Dataset1

\begin{tabular}{lll}
\hline Rank & InCRNA name & PMID \\
\hline 1 & DNM3OS & 27693451 \\
2 & CCAT1 & 31310241 \\
3 & BANCR & 29565494 \\
4 & PANDAR & 26927017 \\
5 & MNX1-AS1 & 30697072 \\
6 & FOXCUT & 25516208 \\
7 & WRAP53 & 26460974 \\
8 & TUG1 & 30098551 \\
9 & MIR17HG & 25680407 \\
10 & IGF2-AS & 33175607 \\
\hline
\end{tabular}

Table 4 Top 10 predicted IncRNAs associated with colon cancer on Dataset1

\begin{tabular}{lll}
\hline Rank & IncRNA name & PMID \\
\hline 1 & UCA1 & 30652355 \\
2 & GAS5 & 27951730 \\
3 & PVT1 & 30504754 \\
4 & SNHG16 & 31502038 \\
5 & XIST & 29679755 \\
6 & DNM3OS & Unconfirmed \\
7 & TUG1 & 27634385 \\
8 & IGF2-AS & 28534511 \\
9 & HULC & 30551459 \\
10 & SPRY4-IT1 & Unconfirmed \\
\hline
\end{tabular}

\section{Case studies}

To further evaluate the capability for detecting unknown lncRNA-disease associations of VGAELDA, case studies were adopted. We predicted the unknown diseaserelated lncRNAs of some specific diseases on the datasets, which can be validated by PubMed literature. The unknown disease-related lncRNAs of a disease are ranked by VGAELDA-predicted score. In this paper, we adopted case studies on lncRNAs associated with breast cancer and colon cancer.

On Dataset 1, the top 10 VGAELDA-predicted IncRNAs associated with breast cancer and colon cancer were listed in Tables 3 and 4, respectively. PMID denotes the PubMed ID of the supporting literature for the corresponding disease-related IncRNAs detected by VGAELDA. Table 3 indicates that all the top 10 VGAELDApredicted lncRNAs associated with breast cancer have been confirmed by previous literature. Table 4 indicates that 8 of the top 10 VGAELDA-predicted lncRNAs associated with colon cancer have been confirmed as well.

On Dataset 2, the top 10 VGAELDA-predicted IncRNAs associated with breast cancer and colon cancer were listed in Additional files 4 and 5. Additional file 4 demonstrates that 8 of the top 10 VGAELDA-predicted lncRNAs associated with breast cancer have 
been confirmed by previous literature. Additional file 5 demonstrates that 9 of the top 10 VGAELDA-predicted IncRNAs associated with colon cancer have been confirmed.

Breast cancer is the most commonly diagnosed cancer and the main threat of health among females worldwide [45]. VGAELDA has been applied to predict potential lncRNAs related to breast cancer. For instance, DNM3OS downregulates Vitamin D receptor (VDR), and VDR is capable of upregulating Suppressor of fused gene ( $\mathrm{SuFu}$ ), while $\mathrm{SuFu}$ is an inhibitor of progression of breast cancer [46]. CCAT1 promotes proliferation and migration of triple-negative breast cancer cells via downregulating miRNA miR-218 and activating the expression of protein ZFX [47]. BANCR is significantly correlated to the growth of breast cancer cells [48].

Colon cancer is a major malignant cancer in digestive system [45]. Among the top 10 IncRNAs predicted by VGAELDA, UCA1 facilitates the progression of colon cancer through upregulating miRNA miR-28-5p and HOXB3 [49]. It is found that GAS5 is positively correlated to colon cancer as well [50]. Also, previous research suggests that PVT1 can sponge miRNA miR-26b and promote proliferation and metastasis of colon cancer [51].

Besides, we listed the predictions of potential lncRNA-disease associations with respect to all diseases of Dataset1 and Dataset2 in Additional files 6 and 7, respectively.

\section{Discussion}

Previous methods for predicting lncRNA-disease associations modeled dependent relationship from features based on some handcrafted measurements of similarity, then propagated labels of samples on the graph constructed via feature similarities. However, it is difficult for those measurements to capture similarities among high-dimensional features directly. Hence, the hyperparameters in these measurements would significantly affect the performance of prediction, which decreases the preciseness of label propagation.

To address this issue, VGAELDA designed representation learning framework that fuses the feature inference network and the label propagation network, to solve graph semi-supervised learning Problem 1 (see Methods). Our Assumption 1 (see Methods) clarifies the capability of an autoencoder to obtain low-rank solution. Based on Assumption 1, an autoencoder with manifold loss as we defined in Definition 1 (see Methods), is competent to obtain the optimal solution of geometric matrix completion problem. Considering the manifold constraint and low-rank constraint that the lncRNA-disease association matrix should satisfy, we adopted VGAE to implement feature inference network GNNq, and GAE to implement label propagation network GNNp. With the alternate training via variational EM algorithm, two GAEs with manifold loss to measure the smoothness of manifold, would significantly strengthen the robustness and preciseness of label propagation through the representations learned by VGAE. Hence the feature similarities, i.e. the topological relationship of the graph, only need to be estimated roughly. The experiments demonstrate that VGAELDA outperforms various kinds of matrix completion based or manifold regularization based methods.

Furthermore, VGAELDA provides an efficient way to integrate information from lncRNA space and disease space. By applying co-training loss as we defined in 
Definition 2 (see Methods), information from lncRNA space and disease space are captured collaboratively. Finally, the association matrix $F_{l}$ computed from lncRNA space and $F_{d}$ computed from disease space, can be integrated simply, since Assumption 1 suggest that both $F_{l}$ and $F_{d}$ follow low-rank property.

\section{Conclusion}

The prediction of potential lncRNA-disease associations is of great importance to disease prognosis, diagnosis and treatment. In this paper, we proposed a deep learning model, VGAELDA, which integrates variational inference and graph autoencoders to detect potential lncRNA-disease associations. VGAELDA designed a representation learning framework to fuse the feature inference network and the label propagation network. Specifically, VGAELDA adopts variational graph autoencoder GNNq for feature inference, and graph autoencoder GNNp for label propagation. These two graph autoencoders are trained alternately in end-to-end manner via variational EM algorithm. This has significantly improved the efficiency of feature representation learning and label propagation. Further discussion demonstrates the validity of VGAELDA to find an optimal solution to the geometric matrix completion problem, and to integrate information from both lncRNA space and disease space. Experiments illustrate that VGAELDA is superior to the current state-of-the-art prediction methods, and case studies indicate that VGAELDA is competent in detecting potential lncRNA-disease associations. The results of evaluation demonstrate that VGAELDA is competent to capture efficient lowdimensional representations from high-dimensional features of both lncRNAs and diseases, and predict unknown lncRNA-disease associations robustly and precisely.

Compared to previous lncRNA-disease associations prediction methods, VGAELDA adopts an end-to-end framework based on variational inference in graph neural networks. VGAELDA is a data-driven end-to-end deep learning approach with a high flexibility. Therefore, VGAELDA is competent to be a general model for graph semisupervised learning and association prediction tasks for other biological entities.

\section{Methods}

\section{Problem formulation}

Suppose the number of lncRNAs and diseases are $m$ and $n$ respectively, and $Y_{m \times n}$ denotes the association matrix. $Y_{i j}=1$ if the association between lncRNA $i$ and disease $j$ is known, otherwise $Y_{i j}=0$. An algorithm predicting lncRNA-disease associations requires $Y$ and corresponding feature matrix $X$ as input, then outputs a score for each pair of lncRNA and disease. $F$ denotes the score matrix, $F_{i j} \in[0,1]$, i.e. the prediction result.

In the view of machine learning, an IncRNA-disease pair is labeled if it has been proved to be associated. Usually, there are only few samples labeled in an lncRNA-disease dataset, and the other tremendous amount of associations need to be detected. Therefore, the prediction for lncRNA-disease associations can be viewed as propagating labels to plenty of unlabeled pairs from few labeled ones, which is classified as semi-supervised learning. 


\section{Variational inference for graph semi-supervised learning Graph semi-supervised learning}

Semi-supervised learning is based on manifold assumption [52]. Manifold assumption clarifies that samples are distributed on a manifold, samples with higher feature similarities are closer on the manifold, and tend to share the same labels. The manifold of data can be depicted by graph structure constructed through feature matrix, which leads to graph semi-supervised learning. This type of methods first computes adjacency matrix from features to construct a graph, then propagate labels from labeled samples to unlabeled ones on this graph iteratively $[53,54]$.

Suppose $L$ denotes normalized Laplacian matrix of the graph, minimizing trace $\left(\mathrm{F}^{\mathrm{T}} \mathrm{LF}\right)$ can obtain the label matrix $F$ following manifold assumption [52, 55]. Belkin et al. [7] added this manifold constraint to least square problem, then derived Laplacian regularized least square (LRLS) method

$$
\min _{F}\|F-Y\|_{F}^{2}+\eta \operatorname{trace}\left(\mathrm{F}^{\mathrm{T}} \mathrm{LF}\right)
$$

where $\|\cdot\|_{F}$ denotes Frobenius norm of a matrix, and $\eta$ is a hyperparameter. Eq. (7) is a trade-off between the accuracy based on labeled data, and the smoothness of the manifold. This is classified as manifold regularization [7]. Label propagation follows the framework of manifold regularization as Eq. (7) [53, 54]. Xia et al. [9] derived that association matrix $F$ follows manifold assumption, and can be obtained via solving Eq. (7).

\section{Graph Markov neural networks}

The motivation of VGAELDA is begun with graph semi-supervised learning from probabilistic perspective. Through this perspective, label propagation can be viewed as maximizing $p\left(y_{u} \mid y_{l}, x_{v}\right)$ [56], where $y_{u}$ and $y_{l}$ denote labels from unlabeled and labeled nodes respectively, and $x_{v}$ denotes attributes of objects on the graph. As the number of $y_{u}$ is often much larger than $y_{l}$, it is difficult to maximize $p\left(y_{u} \mid y_{l}, x_{v}\right)$. Qu et al. [36] proposed Graph Markov Neural Networks (GMNN), suggesting that variational inference for graph semi-supervised learning leads to Problem 1.

Problem 1 Variational inference for graph semi-supervised learning adopts the variational distribution $q\left(y_{u} \mid x_{v}\right)$ to approximate $p\left(y_{u} \mid y_{l}, x_{v}\right)$, which leads to optimize evidence lower bound (ELBO)

$$
\mathbb{E}_{q\left(y_{u} \mid x_{v}\right)}\left[\log q\left(y_{u} \mid x_{v}\right)-\log p\left(y_{l}, y_{u} \mid x_{v}\right)\right] .
$$

Remark of Problem 1 is in the Additional file 8. Since labeled and unlabeled samples are observations and latent variables in conditional random field (CRF), and according to Markov property in CRF, the label of an unlabeled node is only related to its neighborhood. Hence, label propagation procedure aggregates messages from neighborhood, which is intrinsically related to graph neural networks [33].

GMNN adopted two GNNs, GNNq and GNNp, to depict $q\left(y_{u} \mid x_{v}\right)$ and $p\left(y_{l}, y_{u} \mid x_{v}\right)$ respectively, since GNNs are successfully adopted in graph semi-supervised learning [33]. Problem 1 can be solved by variational EM (expectation maximization) algorithm 
[57] (see Additional file 8), GNNq and GNNp are trained by variational EM algorithm, which executes the following two steps alternately until convergence.

- E-step: fix GNNp, and train GNNq by attributes of objects, to obtain the pseudolabels,

- M-step: fix GNNq, and input pseudo-labels into GNNp for training.

\section{Geometric matrix completion}

Except for manifold assumption, the association matrix also follows the low-rank assumption that it lies in a smaller subspace, this leads to the matrix completion [8] problem.

$$
\min _{F} \operatorname{rank}(\mathrm{F}) \quad \text { s.t. } \mathcal{P}_{\Omega}(\mathrm{F})=\mathcal{P}_{\Omega}(\mathrm{Y}),
$$

where $\Omega$ is the set of all known lncRNA-disease associations. The projection operator $\mathcal{P}_{\Omega}(\cdot): \mathbb{R}^{m \times n} \rightarrow \mathbb{R}^{m \times n}$ of matrix $M$ is defined as

$$
\mathcal{P}_{\Omega}(M)_{i j}=\left\{\begin{array}{ll}
M_{i j} & (i, j) \in \Omega \\
0 & \text { otherwise }
\end{array} .\right.
$$

Eq. (9) is an NP-hard and nonconvex problem, thus it is usually relaxed as the following convex surrogate

$$
\min _{F}\|F\|_{*}+\mu\left\|\mathcal{P}_{\Omega}(F-Y)\right\|_{F}^{2} .
$$

where $\|\cdot\|_{*}$ denotes nuclear norm, i.e. the sum of singular values of a matrix.

Geometric matrix completion $[19,20]$ incorporates manifold constraint trace $\left(\mathrm{F}^{\mathrm{T}} \mathrm{LF}\right)$ into low-rank constraint, that is to solve

$$
\min _{F}\|F\|_{*}+\mu\left\|\mathcal{P}_{\Omega}(F-Y)\right\|_{F}^{2}+\eta \operatorname{trace}\left(\mathrm{F}^{\mathrm{T}} \mathrm{LF}\right)
$$

\section{VGAELDA}

\section{Method overview}

We proposed our model, VGAELDA, which designed representation learning framework to fuse the feature inference network and the label propagation network, and is trained through variational EM algorithm using GMNN [36] that integrated variational inference and GNN. VGAELDA executes the following two steps alternately until convergence.

- E-step (feature inference): fix GNNp, and train GNNq by high-dimensional features, to obtain low-dimensional representations,

- M-step (label propagation): fix GNNq, and input lncRNA-disease association matrix into GNNp for training. 


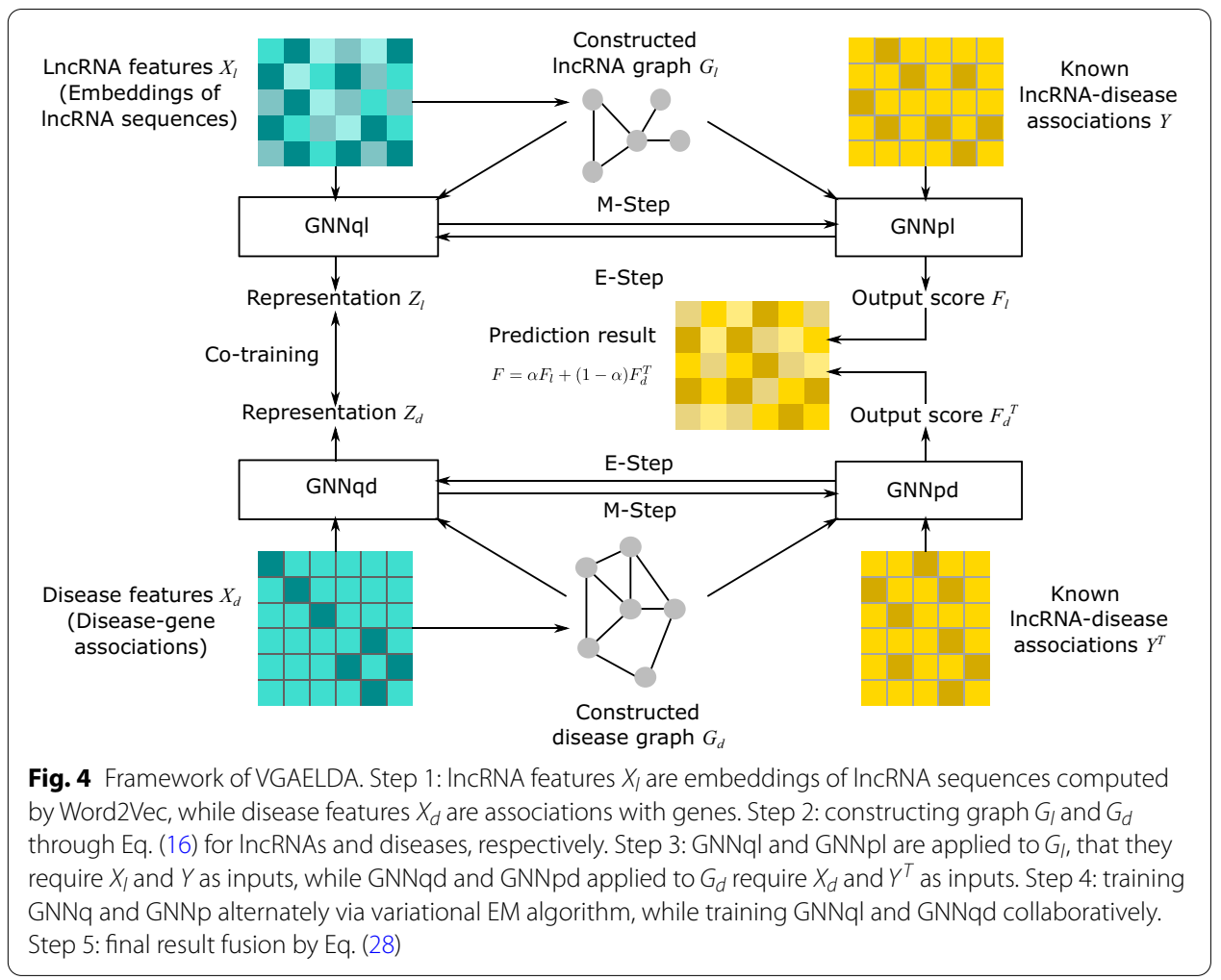

In VGAELDA, feature inference network GNNq is a variational graph autoencoder (VGAE) [37], and label propagation network GNNp is a graph autoencoder (GAE) [37]. Assumption 1 and Definition 1 suggest that the application of these two autoencoders solves the geometric matrix completion problem Eq. (12), for capturing efficient low-dimensional representations via VGAELDA. Furthermore, VGAELDA adopts co-training [58] that integrates information from lncRNA space and disease space. The framework of our model is shown on Fig. 4.

\section{Implementing graph autoencoders}

Each layer of a graph autoencoder is graph convolutional layer. The formula of the $l$-th $(l>0)$ graph convolutional [33] layer is

$$
H^{(l)}=\rho\left(\tilde{D}^{-1 / 2} \tilde{A} \tilde{D}^{-1 / 2} H^{(l-1)} \Theta^{(l)}\right),
$$

where $\tilde{A}$ is adjacency matrix with self-loop, i.e. $\tilde{A}=A+I . \tilde{D}$ is a diagonal matrix called degree matrix, $\tilde{D}_{i i}=\sum_{j} \tilde{A}_{i j}, \rho(\cdot)$ denotes nonlinear activation function, $\Theta^{(l)}$ denotes weight of the $l$-th layer of network, and $H^{(0)}$ is the initial input feature matrix.

Assumption 1 Autoencoder GNNp with $Y$ as input and $F$ as output can obtain the optimal solution of Eq. (11).

Definition 1 (manifold loss) Suppose $Z$ and $Z^{\prime}$ are representations of autoencoder GNNq and GNNp, respectively, then, to optimize manifold constraint trace $\left(\mathrm{F}^{\mathrm{T}} \mathrm{LF}\right)$ can be viewed as optimizing the following manifold loss 


$$
L_{m}=\frac{1}{2}\left\|Z-Z^{\prime}\right\|_{F}^{2}
$$

Remarks of Assumption 1 and Definition 1 are in Additional file 8. In the view of the alternating direction method of multipliers (ADMM) [59], solving the geometric matrix completion problem Eq. (12) can be viewed as optimizing Eq. (7) and Eq. (11) alternately. Therefore, autoencoder GNNp with the addition of manifold loss as we defined in Definition 1, obtains the solution of Eq. (12).

However, to enhance the efficiency of adding manifold loss Eq. (14), we implemented a variational graph autoencoder as GNNq to capture representation $Z$. Suppose the feature matrix of the graph is $X$, the encoder learns mean $\mu$ and standard deviation $\sigma$. The representation $Z$ can be computed by applying reparameterization trick [60], which means

$$
Z=\mu+\sigma \epsilon,
$$

where $\epsilon$ is sampled from standard Gaussian distribution. Then, the decoder reconstructs a feature matrix $X^{\prime}$.

The adjacency matrix of graph $G$ can be constructed simply in this way. Firstly, sort the Euclidean distances among different feature vectors of nodes. Secondly, for each node $i$, select the 10-nearest nodes except itself. Thirdly, suppose the set of these nodes for node $i$ is $\mathcal{N}(i)$, matrix $C$ satisfies that $C_{i j}=1$ if $j \in \mathcal{N}(i)$, otherwise $C_{i j}=0$. The adjacency matrix with self-loop of the constructed graph $G$ is

$$
\tilde{A}=C^{T} \odot C+I,
$$

where $\odot$ denotes Hadamard product.

Network structures of GNNq and GNNp are shown on Additional file 9. As shown on Additional file 9, GNNp is a basic GAE that takes initial label matrix $Y$ as input, the dimension of hidden vector is 256 , output of hidden layer is $Z^{\prime}$, and output of decoder is prediction $F$. GNNq is a VGAE, that each layer of the variational autoencoder [60] is a graph convolutional layer, the dimension of output vectors of each hidden layers in GNNq are 256.

\section{Variational EM algorithm}

The variational EM algorithm is implemented through minimizing the losses of GNNq and GNNp alternately. Similar to other variational graph autoencoders, the loss function of GNNq is the sum of reconstruction error $L_{q r}$, and KL divergence $L_{K L}$.

$$
L_{q}=L_{q r}+L_{K L} .
$$

Kingma and Welling [60] derived that in a variational autoencoder:

- If the features follow Gaussian distribution, the reconstruction error is mean square error. 


$$
L_{q r}=\frac{1}{2}\left\|X-X^{\prime}\right\|_{F}^{2},
$$

- If the features follow Bernoulli distribution, the reconstruction error is cross entropy loss.

$$
L_{q r}=-\sum_{i, j} X_{i j} \log X_{i j}^{\prime}
$$

- KL divergence loss can be computed through

$$
L_{K L}=-\sum_{i, j} \frac{1}{2}\left(1+2 \log \sigma_{i j}-\mu_{i j}^{2}-\sigma_{i j}^{2}\right) .
$$

In VGAELDA, the features of IncRNAs are computed from sequences by Word2Vec [39], and features of diseases are computed through associations with disease-related genes. Thus, lncRNA features follow Gaussian distribution, and disease features follow Bernoulli distribution. Therefore, $L_{q r}$ in GNNql and GNNqd are computed by Eq. (18) and Eq. (19), respectively.

The outputs of encoder and decoder are scaled into $(0,1)$ through applying sigmoid activation function. Meanwhile, following Eq. (7) , the loss function of GNNp is the sum of reconstruction error and manifold loss.

$$
L_{p}=L_{p r}+\gamma L_{m} .
$$

The reconstruction error of GNNp is the cross entropy between prediction and true label

$$
L_{p r}=-\sum_{i, j} Y_{i j} \log F_{i j} .
$$

Then, $F$ is obtained after adopting variational EM algorithm to train GNNq and GNNp alternately until convergence, and is finally scaled into interval $[0,1]$ by

$$
F_{i j} \leftarrow \frac{F_{i j}-F_{\min }}{F_{\text {max }}-F_{\min }},
$$

where $F_{\min }$ and $F_{\max }$ denote minimum and maximum element in matrix $F$.

\section{Integrating information from IncRNA space and disease space}

As shown on Fig. 4, the constructed lncRNA graph $G_{l}$ and disease graph $G_{d}$ are different. Eq. (17) and Eq. (21) can compute loss from $G_{l}$ and $G_{d}$ respectively, but it is important to integrate the information capturing from lncRNA space and disease space. Therefore, we adopt co-training [58] to train GNNql and GNNqd collaboratively.

Definition 2 (co-training loss) Suppose $Z_{l}$ and $Z_{d}$ are representations learned from lncRNA space and disease space, respectively, then co-training loss

$$
L_{c}=\frac{1}{2}\left\|Z_{l} Z_{d}^{T}-Y\right\|_{F}^{2} .
$$


can measure the performance of co-training.

Remark of Definition 2 is in Additional file 8. Then GNNql and GNNqd are trained simultaneously by optimizing the total loss of GNNq

$$
\mathcal{L}_{q}=\alpha L_{q l}+(1-\alpha) L_{q d}+\beta L_{c},
$$

where $L_{q l}$ and $L_{q d}$ denote losses of GNNql and GNNqd computed through Eq. (17) respectively, and $\alpha \in(0,1)$ is the weight parameter that balances information capturing from lncRNA space and disease space. Similarly, the total loss of GNNp is

$$
\mathcal{L}_{p}=\alpha L_{p l}+(1-\alpha) L_{p d},
$$

where $L_{p l}$ and $L_{p d}$ denote losses of GNNpl and GNNpd computed through Eq. (21) respectively. Then, the variational EM algorithm is implemented through optimizing $\mathcal{L}_{q}$ and $\mathcal{L}_{p}$ alternately. After training procedure, GNNpl outputs $F_{l}$ while GNNpd outputs $F_{d}$. Since both $F_{l} \in \mathbb{R}^{m \times n}$ and $F_{d} \in \mathbb{R}^{n \times m}$ are low-rank provided by autoencoders, and through the rank-sum inequality that

$$
\operatorname{rank}\left(\mathrm{aF}_{1}+\mathrm{bF}_{\mathrm{d}}^{\mathrm{T}}\right) \leq \operatorname{rank}\left(\mathrm{F}_{1}\right)+\operatorname{rank}\left(\mathrm{F}_{\mathrm{d}}^{\mathrm{T}}\right), \forall \mathrm{a}, \mathrm{b},
$$

the final result

$$
F=\alpha F_{l}+(1-\alpha) F_{d}^{T} .
$$

is low-rank.

The procedure of VGAELDA is summarized in Algorithm 1, where $X^{\prime}, Z \leftarrow \mathrm{GNN}(\mathrm{G}, \mathrm{X})$ summarizes the computing procedure of a GAE.

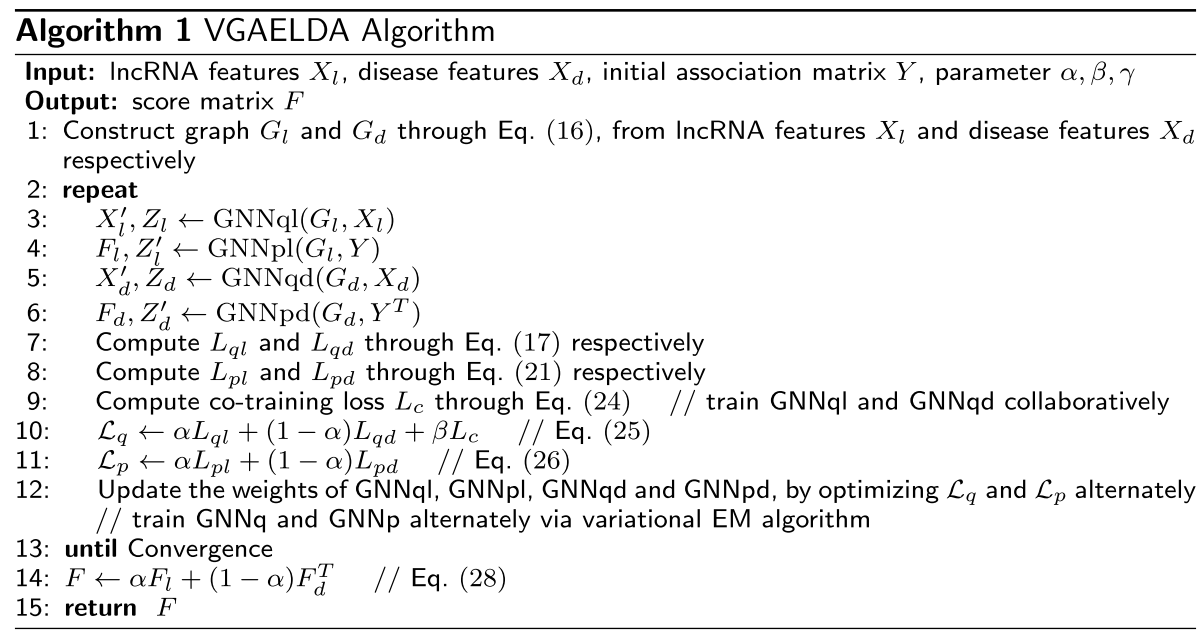

\section{Hyperparameters tuning}

In VGAELDA, there are three hyperparameters, $\alpha, \beta$ and $\gamma$, that need to be tuned. Hyperparameter $\alpha$ depicts a balance between lncRNA space and disease space. However, 
after evaluating our model at each $\alpha \in\{0.1,0.3,0.5,0.7,0.9\}$, we found that VGAELDA is robust to the choice of $\alpha$, and the results are shown on Additional file 10. Hence we simply set $\alpha=0.5$.

Since manifold loss $L_{m}$ and co-training loss $L_{c}$ depend on the computation of representations of GNNql and GNNqd, the capabilities of manifold constraint and co-training constraint are related to the effectiveness of representation capturing by GNNq. Hence, we need to set hyperparameter $\beta$ in Eq. (25) and $\gamma$ in Eq. (21), increasing as training goes, to enhance the robustness of representation learning, and the convergence of EM algorithm. So here we set $\beta=\gamma=e / e_{n}$ at $e$-th epoch, where $e_{n}=500$ denotes the number of epochs.

We adopted PyTorch [61] (https://pytorch.org/) to construct VGAELDA, and applied Adam optimizer [62], where learning rate is 0.01 , weight decay is $10^{-5}$, and we set dropout $=0.5$ [63]. Our model was trained on a single NVIDIA GeForce GTX 2070 GPU with 8GB memory. we evaluated the performance of VGAELDA through varying learning rate in $\{0.001,0.01,0.1,1\}$, and the results are shown on Additional file 11. The figure depicts that the best value of learning rate is 0.01 .

Moreover, we evaluated our model at different dimension of hidden vectors, and the results are shown on Additional file 12. The figure depicts that the performance of our model is enhanced with the increase of hidden vector dimension. However, when the dimension is more than 256, there is little increment and the performance remains stable. Hence, we set the hidden vector dimension at 256 to save the time and space cost of our model.

Besides, we also evaluated our model at different dimension of lncRNA embedding vectors adopted by Word2Vec, and the results are shown on Additional file 13. The figure shows that a larger dimension of lncRNA embedding vectors tends to perform better. However, when the dimension is more than 150, there is little increment and the performance remains stable. Hence, we simply set the dimension of lncRNA embedding vectors at 300 .

\section{Abbreviations}

Acc: Accuracy; AUPR: Area under precision-recall curve; AUROC: Area under ROC curve; EM: Expectation maximization; FN: False negative; FP: False positive; GAE: Graph autoencoder; GCN: Graph convolutional networks; GNN: Graph neural networks; Mcc: Matthews correlation coefficient; LDA: IncRNA-disease association; LncRNA: Long non-encoding RNA; LOOCV: Leave-one-out cross validation; LRLS: Laplacian regularized least square method; Pre: Precision; ROC curve: Receiver operating characteristic (ROC) curve; Sn: Sensitivity; Sp: Speciality; TN: True negative; TP: True positive; VGAE: Variational graph autoencoder.

\section{Supplementary Information}

The online version contains supplementary material available at https://doi.org/10.1186/s12859-021-04073-z.

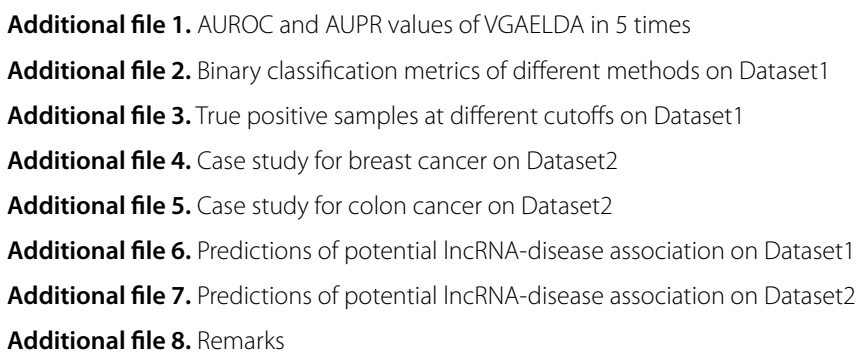


Additional file 9. Network structures

Additional file 10. AUPR at different $a$

Additional file 11. AUPR at different learning rate

Additional file 12. AUPR at different dimension of hidden vectors

Additional file 13. AUPR at different dimension of embedding vectors of IncRNA

\section{Acknowledgements}

Not applicable.

\section{Authors' contributions}

Han Zhang conceived the research. Zhuangwei Shi, Han Zhang, Chen Jin, Xiongwen Quan and Yanbin Yin designed the research. Zhuangwei Shi and Chen Jin implemented the research. Zhuangwei Shi, Han Zhang, Chen Jin and Yanbin Yin wrote the manuscript. All authors read and approved the final manuscript.

\section{Funding}

This research was funded by the National Natural Science Foundation of China Grant No. 61973174.

\section{Availability of data and materials}

All the data using in our paper are collected from the following public datasets. Dataset1 can be downloaded from https://github.com/USTC-HIlab/TPGLDA. Dataset2 can be downloaded from http://mlda.swu.edu.cn/codes.php?name= MFLDA. Both of them were collected from LncRNADisease Database (http://www.cuilab.cn/Incrnadisease). In VGAELDA, the information of IncRNA sequences was downloaded from the Nucleotide Database of NCBI (https://www.ncbi.nlm. nih.gov/nuccore), and the information of diseases was downloaded from DisGeNet (https://www.disgenet.org/home/) and Disease Ontology (https://disease-ontology.org/). The source code is available at https://github.com/zhanglabNKU/ VGAELDA.

\section{Declarations}

\section{Ethics approval and consent to participate}

Not applicable.

\section{Consent for publication}

Not applicable.

\section{Competing interests}

The authors declare that they have no competing interests.

\section{Author details}

${ }^{1}$ College of Artificial Intelligence, Nankai University, Tongyan Road, 300350 Tianjin, China. ${ }^{2}$ College of Computer Science, Nankai University, Tongyan Road, 300350 Tianjin, China. ${ }^{3}$ Department of Food Science and Technology, Nebraska Food for Health Center, University of Nebraska-Lincoln, 1400 R Street, Lincoln, NE 68588, USA.

Received: 28 January 2021 Accepted: 11 March 2021

Published online: 21 March 2021

\section{References}

1. Wapinski O, Chang HY. Long noncoding RNAs and human disease. Trends Cell Biol. 2011;21(6):354-61.

2. Jalali S, Kapoor S, Sivadas A, Bhartiya D, Scaria V. Computational approaches towards understanding human long non-coding RNA biology. Bioinformatics. 2015;31(14):2241-51.

3. Chen X, Yan CC, Zhang X, You Z-H. Long non-coding RNAs and complex diseases: from experimental results to computational models. Brief Bioinform. 2016;18(4):558-76.

4. Sang Y, Tang J, Li S, Li L, Tang X-F, Cheng C, Luo Y, Qian X, Deng L-M, Liu L, Lv X-B. LncRNA PANDAR regulates the g1/s transition of breast cancer cells by suppressing p16(INK4A) expression. Sci Rep. 2016;6:22366.

5. Sun M, Xia R, Jin F, XuT, Liu Z, De W, Liu X. Downregulated long noncoding RNA meg3 is associated with poor prognosis and promotes cell proliferation in gastric cancer. Tumor Biol. 2014;35:1065-73.

6. Faghihi MA, Modarresi F, Khalil AM, Wood DE, Sahagan BG, Morgan TE, Finch CE, St. Laurent III G, Kenny PJ, Wahlestedt C. Expression of a noncoding RNA is elevated in Alzheimer's disease and drives rapid feed-forward regulation of beta-secretase. Nat Med. 2008;14(7):723-30.

7. Belkin M, Niyogi P, Sindhwani V. Manifold regularization: a geometric framework for learning from labeled and unlabeled examples. J Mach Learn Res. 2006;7(1):2399-434.

8. Candès E, Recht B. Exact matrix completion via convex optimization. Found Comput Math. 2009;9(6):717.

9. Xia Z, Wu LY, Zhou X, Wong STC. Semi-supervised drug-protein interaction prediction from heterogeneous biological spaces. BMC Syst Biol. 2010;4(Suppl 2):6.

10. You Z-H, Lei Y-K, Gui J, Huang D-S, Zhou X. Using manifold embedding for assessing and predicting protein interactions from high-throughput experimental data. Bioinformatics. 2010;26(21):2744-51. 
11. Xiao Q, Luo J, Liang C, Cai J, Ding P. A graph regularized non-negative matrix factorization method for identifying microrna-disease associations. Bioinformatics. 2018;34(2):239-48.

12. Chen X, Yan G-Y. Novel human IncRNA-disease association inference based on IncRNA expression profiles. Bioinformatics. 2013;29(20):2617-24.

13. Chen $X$, Yan CC, Luo C, Ji W, Zhang Y, Dai Q. Constructing IncRNA functional similarity network based on IncRNAdisease associations and disease semantic similarity. Sci Rep. 2015;5(1):11338.

14. Xie G, Meng T, Luo Y, Liu Z. SKF-LDA: similarity kernel fusion for predicting IncRNA-disease association. Mol Ther Nucl Acids. 2019;18(6):45-55.

15. Natarajan N, Dhillon IS. Inductive matrix completion for predicting gene-disease associations. Bioinformatics. 2014;30(12):60-8.

16. Chen X, Wang L, Qu J, Guan N-N, Li J-Q. Predicting miRNA-disease association based on inductive matrix completion. Bioinformatics. 2018;34(24):4256-65.

17. Li J, Zhang S, Liu T, Ning C, Zhang Z, Zhou W. Neural inductive matrix completion with graph convolutional networks for miRNA-disease association prediction. Bioinformatics. 2020;36(8):2538-46.

18. Lu C, Yang M, Luo F, Wu F-X, Li M, Pan Y, Li Y, Wang J. Prediction of IncRNA-disease associations based on inductive matrix completion. Bioinformatics. 2018;34(19):3357-64.

19. Kalofolias V, Bresson X, Bronstein MM, Vandergheynst P. Matrix completion on graphs. arXiv preprint. 2014. arXiv: 1408.1717

20. Monti F, Bronstein M, Bresson X. Geometric matrix completion with recurrent multi-graph neural networks. Adv Neural Inf Process Syst. 2017;30:3697-707.

21. Lu C, Yang M, Li M, Li Y, Wu F, Wang J. Predicting human IncRNA-disease associations based on geometric matrix completion. IEEE J Biomed Health. 2018;24(8):2420-9.

22. Wang L, You Z-H, Huang Y-A, Huang D-S, Chan KCC. An efficient approach based on multi-sources information to predict circRNA-disease associations using deep convolutional neural network. Bioinformatics. 2019;36(13):4038-46

23. Xiao Q, Zhang N, Luo J, Dai J, Tang X. Adaptive multi-source multi-view latent feature learning for inferring potential disease-associated miRNAs. Brief Bioinform. 2020.

24. Lan W, Li M, Zhao K, Liu J, Wu F-X, Pan Y, Wang J. LDAP: a web server for IncRNA-disease association prediction. Bioinformatics. 2016;33(3):458-60.

25. Fu G, Wang J, Domeniconi C, Yu G. Matrix factorization-based data fusion for the prediction of IncRNA-disease associations. Bioinformatics. 2017;34(9):1529-37.

26. Ding L, Wang M, Sun D, Li A. TPGLDA: novel prediction of associations between IncRNAs and diseases via IncRNAdisease-gene tripartite graph. Sci Rep. 2018;8(1):1065.

27. Yao D, Zhan X, Zhan X, Kwoh CK, Li P, Wang J. A random forest based computational model for predicting novel IncRNA-disease associations. BMC Bioinform. 2020;21:126.

28. Chen X, Li T-H, Zhao Y, Wang C-C, Zhu C-C. Deep-belief network for predicting potential miRNA-disease associations. Brief Bioinform. 2020.

29. Xuan P, Cao Y, Zhang T, Kong R, Zhang Z. Dual convolutional neural networks with attention mechanisms based method for predicting disease-related IncRNA genes. Front Genet. 2019;10:416.

30. Sheng $\mathrm{N}$, Cui H, Zhang T, Xuan P. Attentional multi-level representation encoding based on convolutional and variance autoencoders for IncRNA-disease association prediction. Brief Bioinform. 2020;1-14.

31. Scarselli F, Gori M, Tsoi AC, Hagenbuchner M, Monfardini G. The graph neural network model. IEEE Trans Neural Netw. 2009;20(1):61-80

32. Xuan P, Pan S, Zhang T, Liu Y, Sun H. Graph convolutional network and convolutional neural network based method for predicting IncRNA-disease associations. Cells. 2019;8(9):1012.

33. Kipf TN, Welling M. Semi-supervised classification with graph convolutional networks. In: Proceedings of the international conference on learning representations (ICLR);2017.

34. Berg R, Kipf T, Welling M. Graph convolutional matrix completion. In: Proceedings of KDD;2018.

35. Wu X, Lan W, Chen Q, Dong Y, Liu J, Peng W. Inferring IncRNA-disease associations based on graph autoencoder matrix completion. Comput Biol Chem. 2020;87:107282.

36. Qu M, Bengio Y, Tang J. GMNN: graph Markov neural networks. Proc Mach Learn Res. 2019;97:5241-50

37. Kipf TN, Welling M. Variational graph auto-encoders. In: NeurIPS Workshop on Bayesian Deep Learning;2016.

38. Chen G, Wang Z, Wang D, Qiu C, Liu M, Chen X, Zhang Q, Yan G, Cui Q. LncRNADisease: a database for long-noncoding RNA-associated diseases. Nucleic Acids Res. 2012;41(D1):983-6.

39. Le Q, Mikolov T. Distributed representations of sentences and documents. Proc Mach Learn Res. 2014;32:1188-96.

40. Asgari E, Mofrad MRK. Protvec: a continuous distributed representation of biological sequences. PLoS ONE. 2015;10(11):0141287.

41. Piñero J, Bravo A, Queralt-Rosinach N, Gutiérrez-Sacristán A, Deu-Pons J, Centeno E, García-García J, Sanz F, Furlong LI. DisGeNET: a comprehensive platform integrating information on human disease-associated genes and variants. Nucleic Acids Res. 2016;45(D1):833-9.

42. Schriml LM, Mitraka E, Munro J, Tauber B, Schor M, Nickle L, Felix V, Jeng L, Bearer C, Lichenstein R, Bisordi K, Campion N, Hyman B, Kurland D, Oates CP, Kibbey S, Sreekumar P, Le C, Giglio M, Greene C. Human disease ontology 2018 update: classification, content and workflow expansion. Nucleic Acids Res. 2018;47(D1):955-62.

43. Xu M, Jin R, Zhou Z-H. Speedup matrix completion with side information: application to multi-label learning. In: Advances in neural information processing systems, 2013;2301-2309.

44. Chicco D, Jurman $\mathrm{G}$. The advantages of the Matthews correlation coefficient (mcc) over $\mathrm{f1}$ score and accuracy in binary classification evaluation. BMC Genom. 2020;21:6

45. Bray F, Ferlay J, Soerjomataram I, Siegel R.L, Torre L.A, Jemal A. Global cancer statistics 2018: Globocan estimates of incidence and mortality worldwide for 36 cancers in 185 countries. CA Cancer J Clin. 2018;68(6):394-424.

46. Alimirah F, Peng X, Gupta A, Yuan L, Welsh J, Cleary M, Mehta RG. Crosstalk between the vitamin d receptor (VDR) and miR-214 in regulating SuFu, a hedgehog pathway inhibitor in breast cancer cells. Exp Cell Res. 2016;349(1):15-22. 
47. Han C, Li X, Fan Q, Liu G, Yin J. Ccat1 promotes triple-negative breast cancer progression by suppressing mir-218/zfx signaling. Aging (Albany NY). 2019;11(14):4858-75.

48. Lou K-X, Li Z-H, Wang P, Liu Z, Chen Y, Wang X-L, Cui H-X. Long non-coding RNA BANCR indicates poor prognosis for breast cancer and promotes cell proliferation and invasion. Eur Rev Med Pharmacol Sci. 2018;22(5):1358-65.

49. Cui M, Chen M, Shen Z, Wang R, Fang X, Song B. LncRNA-uca1 modulates progression of colon cancer through regulating the mir-28-5p/hoxb3 axis. J Cell Biochem. 2019;120(5):6926-36.

50. Poursheikhani A, Abbaszadegan MR, Nokhandani N, Kerachian MA. Integration analysis of long non-coding RNA (IncRNA) role in tumorigenesis of colon adenocarcinoma. BMC Med Genomics. 2020;13:108.

51. Zhang R, Li J, Yan X, Jin K, Li W, Liu X, Zhao J, Shang W, Liu Y. Long noncoding RNA plasmacytoma variant translocation 1 (pvt1) promotes colon cancer progression via endogenous sponging mir-26b. Med Sci Monitor. 2018:24:8685-92.

52. Belkin M, Niyogi P. Laplacian eigenmaps and spectral techniques for embedding and clustering. Adv Neural Inf Process Syst. 2002;15:585-91.

53. Zhou D, Bousquet O, Lal TN, Weston J, Schölkopf B. Learning with local and global consistency. Adv Neural Inf Process Syst. 2004;16:321-8.

54. Wang F, Zhang C. Label propagation through linear neighborhoods. IEEE Trans Knowl Data Eng. 2008;20(1):55-67.

55. Johnson R, Zhang T. On the effectiveness of Laplacian normalization for graph semi-supervised learning. J Mach Learn Res. 2007;8(53):1489-517.

56. Wang J, Shen HC, Wang F, Quan L, Zhang C. Linear neighborhood propagation and its applications. IEEE Trans Pattern Anal Mach Intell. 2009;31(9):1600-15.

57. Neal R, Hinton G. A view of the em algorithm that justifies incremental, sparse, and other variants, 1998;355-368. Springer, Dordrecht.

58. Blum A, Mitchell T. Combining labeled and unlabeled data with co-training. In: Proceedings of the annual conference on computational learning theory, vol. 11, pp. 92-100; 1998

59. Boyd S, Parikh N, Chu E, Peleato B, Eckstein J. Distributed optimization and statistical learning via the alternating direction method of multipliers. Found Trends Mach Learn. 2011;3(1):1-122.

60. Kingma DP, Welling M. Auto-encoding variational bayes. In: Proceedings of the international conference on learning representations. 2014; ICLR.

61. Paszke A, Gross S, Massa F, Lerer A, Bradbury J, Chanan G, Killeen T, Lin Z, Gimelshein N, Antiga L, Desmaison A, Kopf A, Yang E, DeVito Z, Raison M, Tejani A, Chilamkurthy S, Steiner B, Fang L, Bai J, Chintala S. Pytorch: an imperative style, high-performance deep learning library. In: Advances in neural information processing systems, 2019;pp. 8026-8037.

62. Kingma DP, Ba JA. A method for stochastic optimization. In: Proceedings of the international conference on learning representations. 2015; ICLR.

63. Srivastava N, Hinton G, Krizhevsky A, Sutskever I, Salakhutdinov R. Dropout: a simple way to prevent neural networks from overfitting. J Mach Learn Res. 2014;15:1929-58.

\section{Publisher's Note}

Springer Nature remains neutral with regard to jurisdictional claims in published maps and institutional affiliations.

- fast, convenient online submission

- thorough peer review by experienced researchers in your field

- rapid publication on acceptance

- support for research data, including large and complex data types

- gold Open Access which fosters wider collaboration and increased citations

- maximum visibility for your research: over $100 \mathrm{M}$ website views per year

At BMC, research is always in progress.

Learn more biomedcentral.com/submissions 